Animal Biometrics 
Santosh Kumar - Sanjay Kumar Singh

Rishav Singh - Amit Kumar Singh

\section{Animal Biometrics}

Techniques and Applications

黛 Springer 


\author{
Santosh Kumar \\ Department of Computer Science \\ and Engineering \\ Dr. SPM IIIT Naya Raipur \\ Naya Raipur, Chhattisgarh \\ India \\ Sanjay Kumar Singh \\ Department of Computer Science \\ Engineering \\ Indian Institute of Technology (B.H.U) \\ Varanasi, Uttar Pradesh \\ India
}

\author{
Rishav Singh \\ Department of Computer Science \\ Engineering \\ Bennett University \\ Greater Noida, Uttar Pradesh \\ India
}

\author{
Amit Kumar Singh \\ Department of Computer Science \\ and Engineering \\ Jaypee University of Information \\ Technology \\ Waknaghat, Solan, Himachal Pradesh \\ India
}

\author{
ISBN 978-981-10-7955-9 ISBN 978-981-10-7956-6 (eBook) \\ https://doi.org/10.1007/978-981-10-7956-6
}

Library of Congress Control Number: 2017964235

(C) Springer Nature Singapore Pte Ltd. 2017

This work is subject to copyright. All rights are reserved by the Publisher, whether the whole or part of the material is concerned, specifically the rights of translation, reprinting, reuse of illustrations, recitation, broadcasting, reproduction on microfilms or in any other physical way, and transmission or information storage and retrieval, electronic adaptation, computer software, or by similar or dissimilar methodology now known or hereafter developed.

The use of general descriptive names, registered names, trademarks, service marks, etc. in this publication does not imply, even in the absence of a specific statement, that such names are exempt from the relevant protective laws and regulations and therefore free for general use.

The publisher, the authors and the editors are safe to assume that the advice and information in this book are believed to be true and accurate at the date of publication. Neither the publisher nor the authors or the editors give a warranty, express or implied, with respect to the material contained herein or for any errors or omissions that may have been made. The publisher remains neutral with regard to jurisdictional claims in published maps and institutional affiliations.

Printed on acid-free paper

This Springer imprint is published by the registered company Springer Nature Singapore Pte Ltd. part of Springer Nature

The registered company address is: 152 Beach Road, \#21-01/04 Gateway East, Singapore 189721, Singapore 


\section{Preface}

Animal biometrics is an emerging research field for representation and detection of visual animal biometric characteristics. It provides quantified methodologies for design and development of efficient animal biometrics-based recognition systems for different species or individual animal. The animal biometrics-based recognition refers to the use of discriminatory morphological characteristics, physiological and behavioral characteristics (face images, body structure, iris, movement pattern, and gait), phenotypic appearance-based features, and visual features (coat pattern of zebra, spot point on penguin's chest, skin patterning behind the gills of each whale shark and muzzle point image pattern of cattle). The phenotype appearance-based features of species or individual depict the major composite of observable and discriminatory feature characteristics of an organism. Further, it includes the morphological characteristics (image pattern), biochemical traits, phenology, behavior and biometric characteristics for detection and representation of species or individual animal, called animal biometrics identifiers or simply animal biometrics, for automatically detecting, representing, and recognizing a species or individual animal. The research field of animal biometrics uses formal methods to represent and detect biometric features, and morphological image pattern, and phenotypic appearances of animals. It can be utilized to recognize and classify species, identify individuals, detects the occurrence of, or variation in, a distinct behavior, as well as to estimate morphological characteristics and their inter-individual variation or intra-individual differences over time. The formal feature representation based methodologies which have been applied to identify and classify the massive classes of different species for identification of individual animal in the given class. Hence, it performs the detection of animal occurrence or variation in the huge inter-individual and intra-individual classes of species.

In the available literature on animal biometrics, the classical animal recognition methodologies are mainly ear-tagging-based animal identification, freeze branding, ear tattoos, ear-tips, or ear-notches, embedding of microchips and hot iron, embedding of transponders in the animal's body for identification, monitoring, and tracking of animals. These classical animal identification methodologies are invasive approaches for the recognition, and verification of individual cattle. Moreover, the classical animal recognition-based approaches are more susceptible to massive 
vulnerability of loss and illegibility. It always leads to more security issues for the protection of cattle or other animals, as reported in various surveys.

Therefore, classical animal recognition methodology is unable to cater a competent level of security to individual cattle. Moreover, this methodology also fails to provide a required level of registration, identification of missed and swapped cattle, reallocation of livestock, and verification of false insurance claims. Furthermore, the classical animal recognition systems are limited in public domain due to the enormous amount of manpower requirements, high cost, and vulnerability of loss. This loss occurs due to duplication, fraudulent, and forging of embedded standard ear tags. However in the state-of-the-art-based animal recognition approaches, different governmental organizations and private animal insurance providers identify and then verify the animals to solve the biggest problem of the false insurance claims by cutting their ear or snatching the embedded label of ear tags or notches from the animal's ear. The duplication, forgery, and fraudulent processes are responsible for the falsification of the labeled ear tags. Therefore, it is tough to recognize and verify the registered insurance animals (owner of cattle) or impostor (non-insurance) animals.

Thus to provide better solutions for identification and verification of false insurance claims, monitoring of livestock, assistance during health management of animals, efficient recognition is required. The efficient recognition also prevents critical diseases and distribution of cattle in the livestock framework. These are thus the major issues of identification and monitoring of animals in the classical animal recognition approaches and traditional livestock framework-based systems. Further, these issues cannot be ignored by various scientists, veterinary professionals, animal experts, and different research communities before contributing their valuable efforts for the design and development of robust, noninvasive, and real-time animal biometrics-based recognition systems.

Therefore, it is strongly required to develop a real-time animal biometrics-based recognition system for identifying and monitoring different species or individual animal.

The animal biometrics is the emerging field and considered more reliable, like robust biometrics characteristics for verification and identification of species or individual animal than traditional animal identification-based methodologies. The animal biometrics-based recognition system can provide better security, higher efficiency, accuracy, cost-effectiveness and increased user convenience for modern livestock monitoring and frameworks. Therefore, the animal biometrics-based recognition system is used and deployed or evaluated in the livestock frameworks of private or government organizations (e.g., national Aadhar card for animals, verification of false insurance claims, analysis and study of the total species populations, monitoring of animal's health, diagnosis of widespread critical diseases) applications. Each animal biometrics identifier has its strength and weakness, and selection of particular biometric characteristics generally depends on the requirements of the applications. Any chosen biometric characteristics can also be compared on the well-defined factors such as universality, distinctiveness, permanence, collectability, performance, acceptability, and circumvention. Due to well-known distinctiveness and immutable properties of animal biometric identifiers over time, 
these are the most widely used identifiers for recognition and verification of animals.

In view of addressing the above challenges, this book emphasizes the progress made in the classical animal identification methodologies for species or individual animal recognition over the past few decades. The authors believe that this book would provide a sound platform for understanding not only the fundamental coherent set of ideas or concept but also the intricate details of this proliferation and a wide range of technologies of animal biometrics, computer vision, and pattern recognition. In addition, the book is also helpful for the senior undergraduate and graduate students, researcher and industry professional working in this area, and other emerging applications demanding recognition and verification of animals.

The eight chapters of the book are organized as follows:

Chapter 1 presents a brief introduction of the animal biometrics followed by the major characteristics, advantages, potential applications, and interdisciplinary relevance of animal biometrics recognition system in the field of ecology. This chapter further includes the general framework of animal biometrics recognition systems along with major components for detection and identification of species or individual animal along with some state-of-the-art animal biometrics recognition systems. Furthermore, the chapter introduces the population distribution of different species, with opportunities, technological challenges, and recommendations for animal biometrics. Finally, the community, communication, data, and tool sharing are also included to provide the better collaboration to encourage the multidisciplinary researches in the field of animal biometrics.

Chapter 2 presents a comprehensive survey on the state of the art in the field of animal biometrics. This chapter provides a brief introduction to the discipline of animal biometrics followed by the classification and identification techniques of species or individual animal. Furthermore, the potential challenges of existing techniques and research communities, tools, and data sharing are also discussed in brief.

Chapter 3 contains an overview of several reported cattle recognition frameworks based on face biometric features of cattle. Further, the authors in this chapter have developed a biometrics-based cattle recognition system for the validation of prepared face image database of cattle for recognition of individual cattle. The proposed recognition system also has been utilized to evaluate the experimental results of cattle face image by applying the existing handcrafted feature descriptor technique and appearance-based feature extraction and representation techniques.

Chapter 4 presents an automatic recognition algorithm of muzzle point image pattern of cattle for the identification of individual cattle, verification of false insurance claims, registration, and its traceability process. The proposed algorithm uses the texture feature descriptors acquired at each Gaussian smoothed level that are combined using fusion weighted sum rule method. With a muzzle point image pattern database of 500 cattle, the proposed algorithm yields the desired level of identification accuracy. In this chapter, the experimental results demonstrate that the identification accuracy performance of the proposed method is found superior to other appearance-based face recognition algorithms. 
Chapter 5 presents a novel framework using hybrid texture feature extraction and classification approaches to identify cattle based on muzzle point image features. Further, the methods characterize the extracted pattern of muzzle point image for better recognition and classification of cattle, and it examines the discriminatory features of muzzle images using texture feature extraction technique and supervised machine learning-based multiclassifier techniques. Furthermore, the proposed approach is validated in this chapter by achieving the state-of-the-art accuracy on muzzle point image database of cattle with standard identification settings.

Chapter 6 presents deep learning-based cattle recognition system. It is proposed to identify the individual cattle using muzzle point image pattern. The deep learning-based feature extraction and representation approaches are applied in this chapter to learn the discriminatory texture feature representation of muzzle point images with limited training dataset. The proposed approach consists of two steps: (1) a deep mixture model to find accurate patch correspondence between muzzle point image patterns and (2) convolution neural network, deep belief network, and stacked denoising auto-encoder-based fusion network to extract the features from muzzle point image pattern. Extensive experimental results illustrate that the proposed deep learning approach outperforms state-of-the-art methods for recognition of cattle on muzzle point image database.

Chapter 7 presents a novel Fisher locality preserving projection-based cattle recognition framework for extraction and representation of cattle identification in real time. In this chapter, the efficacy of proposed muzzle point-based recognition approach for cattle is evaluated under identification settings which yields excellent recognition rate for identifying individual cattle. Further, the approach is also evaluated for the optimum recognition time for enrollment and recognition of cattle on different sizes of cattle image database.

Finally, Chap. 8 explores the emerging trends and future challenges of state-of-the-art animal recognition techniques in brief. It concludes with the findings of this book and draws potential suggestions for the future research.

This book is an extension of Ph.D. thesis of Dr. Santosh Kumar submitted to the Department of Computer Science \& Engineering, IIT (B.H.U.), Varanasi January 2017, under the supervision of Prof. S. K. Singh.

The authors are indebted to numerous colleagues for the valuable suggestions during the entire period of manuscript preparation.

We would also like to thank publishers at Springer, Ms. Suvira Srivastav, Ms. Yeshmeena Bisht, Ms. Nidhi Chandhoke, and Ms. Krati Shrivastav, for their helpful guidance and encouragement during the creation of this book.

We sincerely thank all authors, editors, and publisher whose works have been cited directly/indirectly in this manuscript.

The authors would not justify their work without showing the gratitude to their family members who have always been the source of strength to tirelessly work to accomplish the assignment.

The second author Prof. S. K. Singh also thank his father 'Sri Balwant Singh' and mother 'Mrs. Savitri Singh' for their blessings and encouragement to do something great in life. 
The third author Dr. Rishav Singh also thank his father 'Dr. Virendra Kumar Singh,' mother 'Rina Singh,' and sister 'Dr. Ritika Singh' for sparing time for this work.

The fourth author Dr. Amit Kumar Singh also thank his father 'Sri Shivraj Singh,' mother 'Mrs. Lila Singh,' wife 'Sweta Singh,' and daughters 'Anandi' and 'Anaya' for sparing time for this work.

\section{Special Acknowledgements}

The first author gratefully acknowledges the authorities of Dr. SPM IIIT Naya Raipur, Chhattisgarh, India, for their kind support to come up with this book.

The second author gratefully acknowledges the authorities of IIT (BHU), Varanasi, India, for their kind support to come up with this book.

The third author gratefully acknowledges the authorities of Bennett University, Greater Noida, India, for their kind support to come up with this book.

The fourth author gratefully acknowledges the authorities of Jaypee University of Information Technology, Waknaghat, India, for their kind support to come up with this book.

Naya Raipur, India

Varanasi, India

Greater Noida, India

Waknaghat, Solan, India

October 2017
Dr. Santosh Kumar

Prof. Sanjay Kumar Singh

Dr. Rishav Singh

Dr. Amit Kumar Singh 


\section{Contents}

1 Animal Biometrics: Concepts and Recent Application . . . . . . . . 1

$1.1 \quad$ Introduction $\ldots \ldots \ldots \ldots \ldots \ldots \ldots \ldots \ldots \ldots \ldots \ldots \ldots \ldots$

1.2 Interdisciplinary Relevance of Animal Biometrics .......... 4

1.3 Promising Applications of Animal Biometrics-Based

Recognition Systems. . . . . . . . . . . . . . . . . 5

1.4 Prerequisites for Promising Applications of

Animal Biometrics . . . . . . . . . . . . . . . . 7

1.5 Animal Biometrics Recognition System . . . . . . . . . . . 8

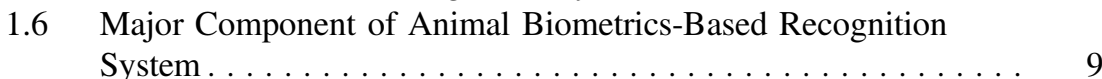

1.6.1 Data Acquisition and Data Preprocessing. . . . . . . . 11

1.6.2 Extraction and Representation of Features . . . . . . . . 11

1.6.3 Matching of Animal Biometric Features ......... 11

1.7 Current State-of-the-Art Animal Biometrics Recognition

Systems . . . . . . . . . . . . . . . . . . . . . 12

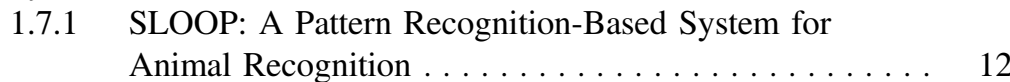

1.7.2 Animal Biometrics-Based Recognition System for Face Detection of Chimpanzee ................. . 14

1.7.3 ECOCEAN Whale Shark Identification System ...... . 15

1.8 Issues and Challenges of Animal Biometrics . . . . . . . . 16

1.8.1 Identification of Animal Based on Coat Pattern . . . . . 16

1.8.2 Segmentation of Background and Discriminatory

Features in Natural Environments . . . . . . . . . . . . 16

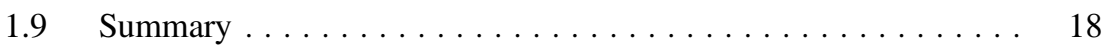

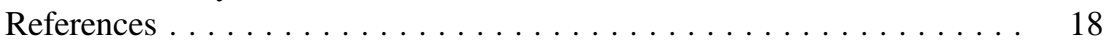

2 Analytical Study of Animal Biometrics: A Technical Survey . . . . . 21

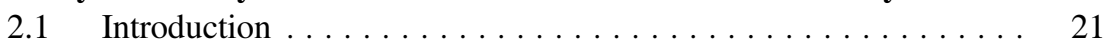

2.2 Classical Animal Identification Methodology .......... 23 
2.2.1 Permanent Identification Methodology . . . . . . . . . . 24

2.2.2 Semipermanent Animal Identification Methodology . . . 27

2.2.3 Temporary Animal Identification Methodology ...... 28

2.3 Visual Animal Biometrics: Current Trends of Animal

Recognition Methodologies . . . . . . . . . . . . . . 32

2.3.1 Retinal Vascular Pattern-Based Cattle Identification .... 32

2.3.2 Iris Pattern-Based Cattle Identification . . . . . . . . . . 34

2.3.3 Cattle Identification Using Biometric Features . . . . . . 35

2.3.4 Muzzle Print Image-Based Cattle Identification . . . . . . 35

2.3.5 Muzzle Point Image Pattern-Based Cattle Identification . . . . . . . . . . . . . . . . . . 39

2.3.6 Identification of Cattle Based on Face Biometric Feature .................... 42

2.4 Morphological Image Pattern-Based Animal Identification. . . . . 43

2.4.1 Identification and Classification of Animal Using

Profiling Behavior ................. 46

2.5 Research Contribution, Communities, Simulation Tools, and Sharing of Database . . . . . . . . . . . . . . . . 58

2.6 Current Trends and Methods . . . . . . . . . . . . . . . 60

2.7 Technical Issues and Challenges $\ldots \ldots \ldots \ldots \ldots \ldots \ldots \ldots 63$

2.7.1 Algorithmic Challenges . . . . . . . . . . . . . . 63

2.7.2 Integration and Unification Structure of Output Data ... 65

2.7.3 Feature Extraction and Representation Challenges ..... 65

2.7.4 Challenges of Feature Extraction and Representation of Species Using Holistic and Texture Feature-Based Techniques ..................... 66

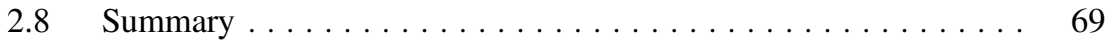

References ............................. 69

3 Recognition of Cattle Using Face Images . . . . . . . . . . . . . . . . 79

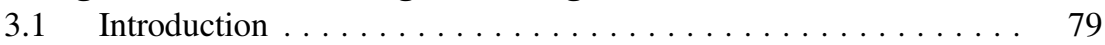

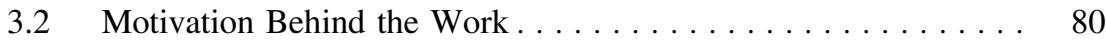

3.3 Preparation and Description of Face Database .......... 82

3.4 Proposed Cattle Recognition System ... . . . . . . . . . 86

3.4.1 Sensor Module (Data Acquisition Phase) . . . . . . . . . 87

3.4.2 Preprocessing and Enhancement Phase ........... 88

3.4.3 Challenges of Face Recognition in Cattle . . . . . . . 89

3.5 Feature Extraction and Matching Phase . . . . . . . . . . 92

3.6 Experimental Result and Discussion . . . . . . . . . . . . . 94

3.6.1 Performance Evaluation . . . . . . . . . . . . . . . . . 94

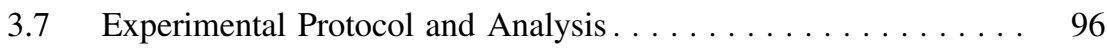

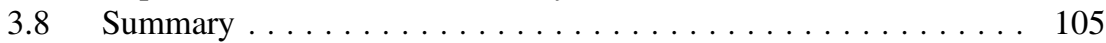

References .............................. 107 


\section{Muzzle Point Pattern-Based Techniques for Individual Cattle}

Identification . . . . . . . . . . . . . . . . . . . . . . . . . . . . . . 111

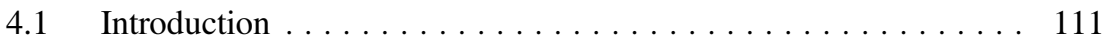

4.1.1 Motivation Behind the Work . . . . . . . . . . . 113

4.1.2 Major Contributions of the Research Work . . . . . . . . 114

4.2 Biometric Characteristics of Muzzle Point Images . . . . . . . . . . 115

4.3 Proposed System . . . . . . . . . . . . . . . . . . 115

4.3.1 Preprocessing and Enhanced of Muzzle Point Image Pattern . . . . . . . . . . . . . . . . . . . . . 117

4.3.2 Image Enhancement Using CLAHE Technique . . . . . . 117

4.3.3 Segmentation and Feature Extraction . . . . . . . . . . 120

4.3.4 Matching of Muzzle Point Image Using

Chi-Square-Based Matching Technique . . . . . . . . . 121

4.4 Experimental Results and Discussion . . . . . . . . . . . . . . 123

4.4.1 Database Preparation and Description . . . . . . . . . . 123

4.4.2 Performance Evaluations of Proposed Algorithm . . . . . . 125

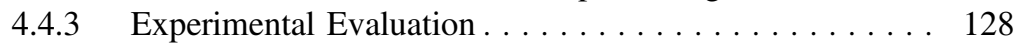

4.5 Summary and Future Directions . . . . . . . . . . . . . 131

References . . . . . . . . . . . . . . . . . . . . . . . . . . . . 132

5 Identification of Cattle Based on Muzzle Point Pattern: A Hybrid

Feature Extraction Paradigm . . . . . . . . . . . . . . . . . . . . . . 137

$5.1 \quad$ Introduction . . . . . . . . . . . . . . . . . . . . . 137

5.1.1 Major Contribution of the Work . . . . . . . . . . . . 140

5.2 Materials and Methods . . . . . . . . . . . . . . . . . . . 140

5.3 Proposed Cattle Recognition System . . . . . . . . . . . . . . . . . 141

5.3.1 Preprocessing and Enhancement Process of Muzzle

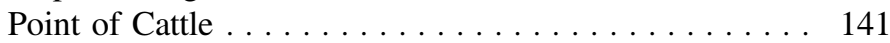

5.3.2 Segmentation of Muzzle Point Image . . . . . . . . . . . . 143

$5.4 \quad$ Feature Extraction and Matching . . . . . . . . . . . . . . . . . . . . 147

5.5 Experimental Results Performance Evaluation . . . . . . . . . . . . 148

5.6 Performance Analysis . . . . . . . . . . . . . . . . . . . . 151

5.7 Recognition of Muzzle Point Image Under

Different Rotations . . . . . . . . . . . . . . . . . . . . . . . . . 154

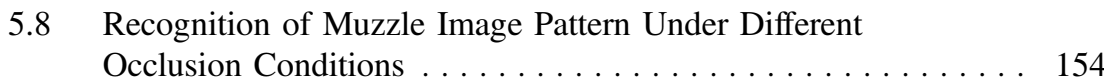

$5.9 \quad$ Summary . . . . . . . . . . . . . . . . . . . . . . 156

References .............................. 158 
6 Deep Learning Framework for Recognition of Cattle Using Muzzle

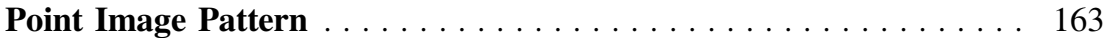

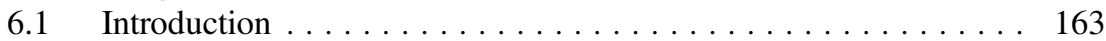

6.1.1 Motivation Behind the Work . . . . . . . . . . . . . 164

6.1.2 Major Research Contributions ................. 165

6.2 Biometric Characteristics of Muzzle Point Image

Pattern of Cattle . . . . . . . . . . . . . . . . . . . 166

6.3 Proposed System . . . . . . . . . . . . . . . . . . 167

6.3.1 Preprocessing and Enhancement . . . . . . . . . . . . 167

6.3.2 Feature Extraction and Representation . . . . . . . . . 168

6.4 Stacked Denoising Auto-encoder Technique.............. 174

6.5 Pretraining and Generalizability of Proposed Recognition Model . . . . . . . . . . . . . . . . . . . . . . . . . . . . . . . . . 177

6.6 Experimental Results and Discussions . . . . . . . . . . . 178

6.6.1 Performance Evaluations. . . . . . . . . . . . . . . . . 178

6.6.2 Comparative Analysis. . . . . . . . . . . . . . . . . . . . 187

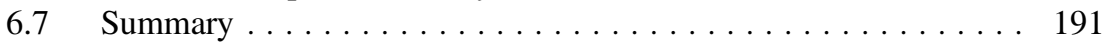

References . . . . . . . . . . . . . . . . . . . . . . . . . 192

7 Real-Time Recognition of Cattle Using Fisher Locality Preserving

Projection Method . . . . . . . . . . . . . . . . . . . . . . . . 197

$7.1 \quad$ Introduction . . . . . . . . . . . . . . . . . . . . . . . . . 197

7.1.1 Contribution Are Illustrated in Brief . . . . . . . . . . . 199

7.2 Real-Time Cattle Recognition System . . . . . . . . . . . . . 200

7.3 Proposed FLPP Feature Extraction and Representation

Approach . . . . . . . . . . . . . . . . . . . . . . . . . . . . . . . 204

7.4 Feature Encoding . . . . . . . . . . . . . . . . 207

7.4.1 Classification Model Using One-Shot Similarity and One-Class-Based SVM . . . . . . . . . . . . . . . . 208

7.4.2 Fishers Linear Discriminant Analysis (FLDA) and OSS Classification Techniques ............. . 209

7.5 Matching of Muzzle Point Images . . . . . . . . . . . . . 210

7.6 Algorithm for Evaluation for Experimental Results . . . . . . . . 212

7.6.1 Appearance-Based and Texture-Based Feature

Extraction Algorithm . . . . . . . . . . . . . . . . 212

7.7 Experimental Result and Analysis . . . . . . . . . . . . . . . 213

7.7.1 Performance Evaluation . . . . . . . . . . . . . . . 213

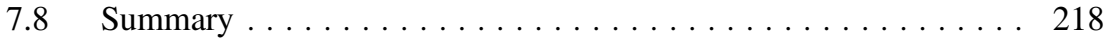

References ............................. 218 


\section{Biometric Methods for Animal: Recent Trends and Future}

Challenges . . . . . . . . . . . . . . . . . . . . . . . . . 223

8.1 Introduction . . . . . . . . . . . . . . . . . . . . 223

8.2 Animal Biometrics-Based Recognition Systems . . . . . . . . . . 225

8.2.1 Low-Cost Cattle Recognition System Using

Multimedia Wireless Network . . . . . . . . . . . 226

8.2.2 Cattle Verification System Using Smart Devices . . . . . 226

8.3 Identification and Monitoring of Pet Animal Using Animal

Biometrics . . . . . . . . . . . . . . . . . . . . . . . . 227

8.4 Horse Identification System Using Animal Biometrics . . . . . . . 230

8.5 Face Recognition Framework for Chimpanzee Using Deep

Learning Approach . . . . . . . . . . . . . . . . . . . . 232

8.6 Shared Tools, Availability of Animal Database. . . . . . . . . . . . 234

8.7 Visual Animal Biometrics: Issues and Challenges . . . . . . . . . . 234

8.8 Summary and Future Directions . . . . . . . . . . . . . . . . . 239

References . . . . . . . . . . . . . . . . . . . . . . . . . . . . . 240 


\section{About the Authors}

Santosh Kumar is an Assistant Professor of Computer Science and Engineering. Prior to joining the Dr. SPM International Institute of Information Technology, Naya Raipur (IIIT Naya Raipur), Chhattisgarh, he was a Ph.D. research scholar in the Department of Computer Science and Engineering, the Indian Institute of Technology (Banaras Hindu University) (IIT (B.H.U.)), Varanasi, Uttar Pradesh, India. He is a member of the Computer Society and the Association for Computing Machinery. His research interests include Animal Biometrics, Computer Vision, Machine Learning, Pattern Recognition, Wireless Sensors, and Internet of Things (IoT).

Sanjay Kumar Singh completed his B.Tech. in Computer Engineering, M.Tech. in Computer Applications, and Ph.D. in Computer Science and Engineering. Currently, he is an Associate Professor in the Department of Computer Science and Engineering, Indian Institute of Technology (Banaras Hindu University), (IIT (BHU)), Varanasi, Uttar Pradesh, India. He is a Certified Novell Engineer (CNE) and Certified Novell Administrator (CNA) from Novell Netware, USA. He is a member of LIMSTE, IEE, the International Association of Engineers, and ISCE. His research areas include Biometrics, Computer Vision, Image Processing, Video Processing, Pattern Recognition, Artificial Intelligence, and Big Data.

Rishav Singh received his B.Tech. from C. V. Raman College of Engineering (CVRCE), Orissa, India, in 2009. He completed his M.Tech. from Jaypee University of Information Technology (JUIT), India, in 2011 and his Ph.D. from the Indian Institute of Technology (Indian School of Mines) (IIT(ISM)), Dhanbad, in 2017. He is currently a member of Infosys Limited. He has extensive IT experience, with proven expertise in the full software development life cycle, including requirement analysis, designing, coding, deploying the code in different environments, and implementation of software applications using R, Cassandra, Machine Learning, Cloudera Hadoop (MapReduce), HBase, and Hive. His research interests include Big Data and Analytics, Machine Learning, Biometrics, and Image Processing. 
Amit Kumar Singh is currently working as an Assistant Professor (Senior Grade) in the Department of Computer Science and Engineering, Jaypee University of Information Technology (JUIT), India. He completed his Ph.D. degree from the Department of Computer Engineering, National Institute of Technology (NIT), Kurukshetra, in 2015. Recently, Dr. Singh was appointed as Associate Editor of IEEE Access and Multimedia Tools and Applications (MTAP), Springer. He has published and presented over 50 research papers in reputed journals and at various national and international conferences. His important research contributions include developing watermarking methods that offer a good trade-off between major parameters, i.e., perceptual quality, robustness, embedding capacity, and the security of watermark embedding into digital images. His research interests include Data Hiding, Biometrics, and Cryptography. 


\section{Abbreviations}

\begin{tabular}{ll} 
AFR & Affordability requirement \\
Batch-CCIPCA & Batch-candid covariance-free incremental-PCA \\
CLAHE & Contrast limited adaptive histogram equalization \\
CMC & Cumulative match characteristic \\
CNN & Convolutional neural network \\
CRS & Cattle recognition system \\
DBN & Deep belief network \\
DT & Decision tree \\
DTM & Data transfer and management \\
EAD & Ease to access database \\
EER & Equal error rate \\
EN & Ear notching \\
EOAS & Ease of application system \\
FAR & False acceptance rate \\
FLDA & Fisher linear discriminant analysis \\
FLLP & Fisher locality preserving projection \\
FMR & False matching rate \\
FNMR & False non-matching rate \\
FRR & False rejection rate \\
GAR & Genuine accept rate \\
GMM & Gaussian mixture model \\
HOOG & Haar of oriented gradient \\
ICA & Independent component analysis \\
ILDA & Incremental linear discriminant analysis \\
IND-CCIPCA & Independent-candid covariance-free incremental-PCA \\
INT & Intermediate \\
K-NN & K-nearest neighbor \\
LBP & Local binary pattern \\
LDA & Linear discriminant analysis \\
LO & Low \\
\hline &
\end{tabular}


LP

LTE

MLP

MO

MPI

NA

OSS

PCA

PFFD

PIM

PNN

PSDFC

RBM

RFID

RIFT

RPCA

RVP

SDAE

SFTA

SIFT

SIM

SOS

SRORD

SURF

SVM

TDL

TIM

UID

VLAD
Lack of pain

Law's texture energy

Multilayer perceptron

Moderate

Muzzle point images

Not available

One-shot similarity

Principal component analysis

Protection from fraud and duplication

Permanent identification methodology

Probabilistic neural network

Protection and security during food chain

Restricted Boltzmann machine

Radio frequency identification

Rotation-invariant feature transformation

Robust principal component analysis

Retinal vascular pattern

Stacked denoising auto-encoder

Segmentation-based fractal texture analysis

Scale-invariant feature transform

Semi-permanent identification methodology

Scalability of system

Success rate of reading database

Speeded-up robust feature

Support vector machine

Tucker tensor decomposition

Temporary identification methodology

Unique identification number

Vector of locally aggregated descriptors 


\section{List of Figures}

Fig. $1.1 \quad$ Coat pattern structure of different species . . . . . . . . . 4

Fig. 1.2 Detection and classification of different species and individual animal using animal biometrics-based

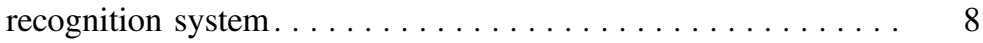

Fig. 1.3 Major components of animal biometrics recognition system . . . . . . . . . . . . . . . . . 10

Fig. 1.4 Major components of SLOOP animal identification system ................. 13

Fig. 1.5 Identification of different species using SLOOP animal

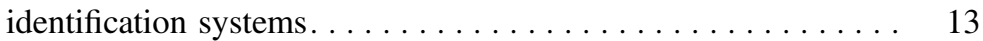

Fig. 1.6 Components of chimpanzee recognition-based systems . . . . . . 14

Fig. 1.7 Detection of keypoints feature of face image of chimpanzee . . . . . . . . . . . . . . . . . . . . . . . . . 14

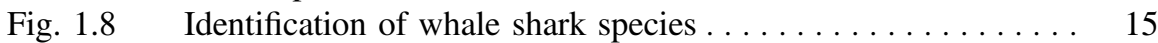

Fig. 1.9 Major components of animal biometrics

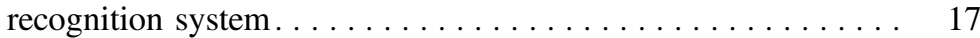

Fig. 2.1 Classical animal identification methods . . . . . . . . . . . . 24

Fig. 2.2 (a) Process of ear-notching process of cattle, (b) tattooing process on ear of cattle, and (c) embedding of ear tag with radio frequency identification (RFID) sensor . . . . . . . 25

Fig. 2.3 Cattle's breed ears have been hurt and disintegrated

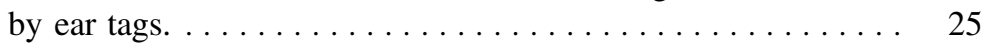

Fig. 2.4 Cattle monitoring and tracking using RFID-based sensor network. . . . . . . . . . . . . . . . . . . . . . 29

Fig. 2.5 Enrollment of retinal image pattern of cattle . . . . . . . . 32

Fig. 2.6 a Enrollment of cattle using retinal pattern, b image of same

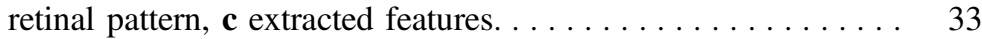

Fig. 2.7 Block diagram of cattle recognition based on muzzle print images of cattle $\ldots \ldots \ldots \ldots \ldots \ldots \ldots \ldots \ldots \ldots \ldots \ldots \ldots$ 
Fig. 2.8 Ridge ending and ridge bifurcation of muzzle point image

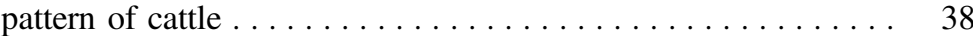

Fig. 2.9 Muzzle point-based cattle identification . . . . . . . . . . . . 40

Fig. 2.10 Flowchart for cattle identification using face image biometric feature . . . . . . . . . . . . . . . . . . . . . 42

Fig. 2.11 Generation of encoded feature using local binary pattern

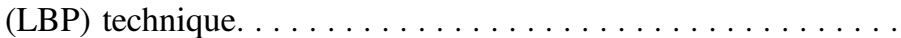

Fig. 2.12 Working of animal biometrics-based recognition system in the various phases and pipeline of local statistical feature-based

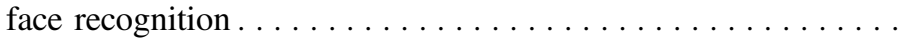

Fig. 2.13 Tortoise identification using local binary pattern-based feature

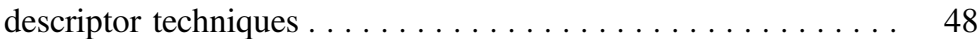

Fig. 2.14 Working prototype model of animal biometrics for identification of species . . . . . . . . . . . . . . . . 62

Fig. 2.15 Issues and challenges of animal biometrics . . . . . . . . 63

Fig. 2.16 Hierarchy of image transformation and feature extraction

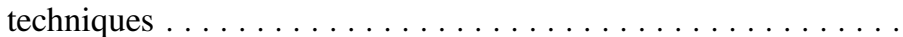

Fig. 2.17 Extraction and representation of feature of

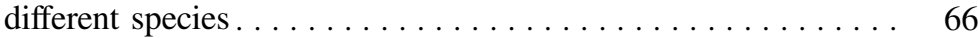

Fig. 3.1 Some face images of cattle from database . . . . . . . . . 83

Fig. 3.2 Inter-class and intra-class variations of face

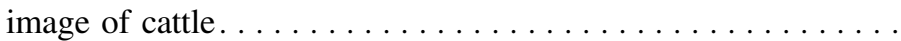

Fig. 3.3 Some challenging face images of cattle from the database . . . . . . . . . . . . . . . . . . . 85

Fig. 3.4 Proposed block diagram of cattle recognition system . . . . . . 87

Fig. 3.5 Computation of mean of face images of cattle......... 93

Fig. 3.6 Computation eigenfaces of face of cattle on (a) and (b) and reconstruction of original face images using eigenvectors.

Fig. 3.7 Between-class and within-class feature scatters in the feature space . . . . . . . . . . . . . . . . . . . . . . 97

Fig. 3.8 Reconstruction of face image of cattle using

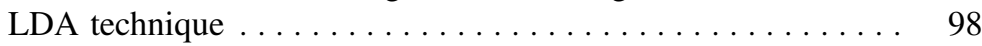

Fig. 3.9 Identification of face image of cattle using linear discriminant-based similarity matching techniques. . . . . . . .

Fig. 3.10 Representation of extracted facial feature of face image of cattle using linear discriminant analysis technique

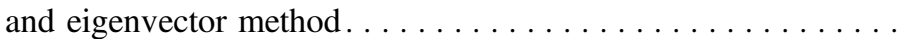

Fig. 3.11 Identification accuracy of PCA, LDA, and ICA technique. . . . . . . . . . . . . . .

Fig. 3.12 To show identification accuracy of batch-LDA, CCIPCA-LiBSVM, ICA-LiBSVM, incremental-LDA (iLDA), and LDA-LiBSVM for cattle face (based on Table 3.4) . 
Fig. 3.13 CMC to show recognition accuracy of cattle

face image based on Table $3.3 \ldots \ldots \ldots \ldots \ldots \ldots$

Fig. 4.1 Beads and ridges features of the muzzle point image pattern of cattle from the database. . . . . . . . . . . . . . 115

Fig. 4.2 Steps involved in the muzzle point pattern recognition

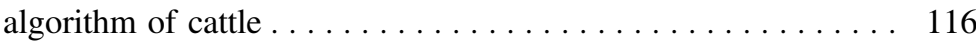

Fig. 4.3 Preprocessing and image enhancement process of muzzle

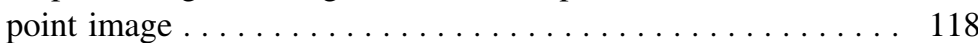

Fig. 4.4 Preprocessing of muzzle point image of cattle. . . . . . . . 118

Fig. 4.5 Illustrates the preprocessing and extraction of bead and ridge feature from the muzzle point image of cattle . . . . . . . . 119

Fig. 4.6 Illustration of segmentation and feature extraction process for muzzle point images pattern of cattle. . . . . . . . . . . . . . 120

Fig. 4.7 Sample image of muzzle point pattern of cattle from database . . . . . . . . . . . . . . . . . . . . . 124

Fig. 4.8 Some challenging images from the cattle database . . . . . . 125

Fig. 5.1 Block diagram of the proposed automated identification system of cattle based on muzzle pattern. . . . . . . . . . 139

Fig. 5.2 Preprocessing: a Original image, b blurred image, $\mathbf{c}$ enhanced muzzle point images . . . . . . . . . . . . . . . . . 142

Fig. 5.3 Preprocessing of muzzle point image of cattle. . . . . . . . . . 142

Fig. 5.4 a Original image and b segmented image of muzzle image pattern

Fig. 5.5 a Original muzzle point image pattern, b segmented muzzle image with color K-means-based segmentation approach

Fig. 5.6 Segmentation of muzzle point image pattern: a Original muzzle images, ground truth, and $\mathbf{b}-\mathbf{e}$ found out ROI using texture segmentation algorithm

Fig. 5.7 Segmentation of muzzle point image pattern using K-means color cluster algorithm.

Fig. 5.8 Watershed-based segmentation of muzzle point image: a-b Selection of region of interest (ROI) from muzzle point image pattern database . . . . . . . . . . 147

Fig. 5.9 Distribution of muzzle point image database . . . . . . . . . . 150

Fig. 5.10 Sample images of muzzle point pattern are rotated with different angles $\left(0^{\circ}, 45^{\circ}, 90^{\circ}, 135^{\circ}\right.$, $180^{\circ}, 225^{\circ}, 270^{\circ}$, and $315^{\circ}$ )

Fig. 5.11 Samples of occluded muzzle images, top row $\mathbf{a}-\mathbf{d}$ represents bottom (horizontal) occlusion, and bottom row $\mathbf{e}-\mathbf{h}$ represents top (vertical) occlusion 
Fig. 6.1 Preprocessing process: a muzzle point image, b filtration of discriminatory features (beads and ridges), $\mathbf{c}$ the removal of background, $\mathbf{d}$ find out the ridges and bead features from the

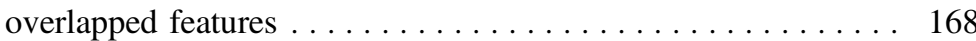

Fig. 6.2 Extraction of discriminatory feature (bead and ridge pattern features) from muzzle point image. . . . . . . . . . . . . . 168

Fig. 6.3 Segmentation of muzzle point images: a the region of interest (ROI) of muzzle point images, b ridge bifurcation (green color) and ridge termination (red color) are extracted from ROI, and $\mathbf{c}$ suppression of irrelative feature information . . . . .

Fig. 6.4 Block diagram of proposed deep learning-based framework for cattle recognition . . . . . . . . . . . . . . . . . . . . . . 169

Fig. 6.5 Steps of proposed deep learning-based framework for cattle recognition using convolution neural network (CNN) technique .

Fig. 6.6 Deep learning architecture of DBN deep learning model composed of four stacked RBM classification approaches

Fig. 6.7 a $20 \%$ corrupted images of muzzle point pattern and b $30 \%$ corrupted muzzle point images using stacked denoising auto-encoder

Fig. 6.8 SIFT keypoints descriptor: (a) input muzzle point image, (b) a SIFT descriptor of the size $(m \times n)$ is chosen from muzzle point image, (c) selection of $16 \times 16$ pixel orientations, (d) $4 \times 4$ cell descriptor with 8 pixel orientations is chosen. The size of single SIFT keypoints feature descriptor is $4 \times 4 \times 8=128$ element . . . . . . . . . . . . . . . .

Fig. 6.9 a The process of SIFT keypoints localization and detection and $\mathbf{b}$ matching of test muzzle point image with stored muzzle point image using SIFT keypoints descriptor. . . . . . . . .

Fig. 6.10 Extraction and encoding of local binary pattern-based descriptor features from the muzzle point image pattern . . . . . .

Fig. 6.11 Process of building of SURF descriptor: $\mathbf{a}, \mathbf{b}$ the detection of keypoints in the muzzle point images, c matching of muzzle point images based on keypoints SURF descriptor with size (for a neighborhood of size $6 \mathrm{X} \mathrm{s}$ where $\mathrm{s}$ is scaling parameter of wavelet responses in horizontal and vertical directions) . . . . 180

Fig. 6.12 Matching of keypoints of muzzle point images using SURF descriptor technique. . . . . . . . . . . . . . . . 180

Fig. 6.13 CMC curve illustrates the identification accuracy versus number of features . . . . . . . . . . . . . . . . . . . . . . . . . . 182

Fig. 6.14 CMC curve illustrates the identification accuracy versus number of patches (images) . . . . . . . . . . . . . . . . 182

Fig. 7.1 Block diagram for transferring the cattle images to server. . . . 200 
Fig. 7.2 Block diagram model of proposed cattle recognition system . . . . . . . . . . . . . . . . . 201

Fig. 7.3 Segmentation process of muzzle point image . . . . . . . . . 203

Fig. 7.4 a Input muzzle point images $\mathbf{b}$ texture feature-based segmentation algorithm . . . . . . . . . . . . . . . . . . 204

Fig. 7.5 Feature points of 10 different pixel values of muzzle classes. . . . . . . . . . . . . . . . . . . . . . . . 207

Fig. 7.6 Feature extraction and representation using FLLP approach . . . . . . . . . . . . . . . . . . . . . . 207

Fig. 7.7 Matching of muzzle point images using one-shot similarity

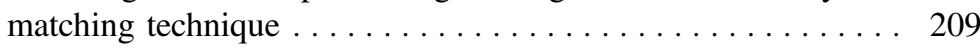

Fig. 7.8 Minutiae feature points of muzzle images of cattle . . . . . . 211

Fig. 7.9 Finding of the region of interest of muzzle points . . . . . . . 211

Fig. 7.10 Identification accuracy of cattle based on number of muzzle point features . . . . . . . . . . . . . . . . . 214

Fig. 7.11 Identification rates $(\%)$ based on muzzle point features . . . . . 214

Fig. 7.12 Identification accuracy (\%) of cattle using Batch-ILDA, CCIPCA-LiBSVM, ICA-LiBSVM, incremental-LDA, and ILDA-LiBSVM.

Fig. 7.13 Illustrates the impact of recognition time $(t)$ of cattle recognition system on the different sizes $(\mathrm{kB})$ of muzzle point

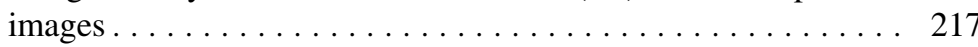

Fig. 8.1 Low-cost cattle recognition system using multimedia wireless network ........................ 226

Fig. 8.2 Proposed animal biometrics-based recognition system

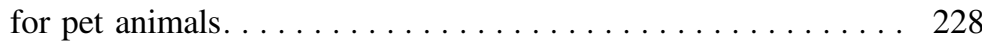

Fig. 8.3 Major steps of proposed framework for pet animals . . . . . . 229

Fig. 8.4 Deep learning-based face recognition model for chimpanzee ...................... 233

Fig. 8.5 Major issues and challenges of visual animal biometrics for identification and classification of species . 


\section{List of Tables}

Table 1.1 Detail description of identification and verification systems for animals ...................... 6

Table 2.1 Comparative analysis of cattle identification ........... 30

Table 2.2 Comparison of classical identification methods for animals.... 31

Table 2.3 Comparative study and cost estimation of various classification approaches .................. 31

Table 2.4 Identification of species based on morphological image features ........................ 44

Table 2.5 Computer vision and pattern recognition techniques ....... 49

Table 2.6 Complete descriptions of computer vision techniques for identification of species . . . . . . . . . . . . . 52

Table 2.7 Classification of species based on animal behavior . . . . . . 55

Table 2.8 Ongoing projects of animal biometrics . . . . . . . . . . 59

Table 2.9 Comparative study of holistic and texture feature-based feature extraction and representation techniques ........ 68

Table 3.1 Description of face image database of cattle .......... 83

Table 3.2 Identification accuracy (\%) of face images of cattle using PCA, LDA, and ICA approaches................. 100

Table 3.3 Identification accuracy (\%) of face images of cattle using batch-ILDA, CCIPCA-LiBSVM, ICA-LiBSVM, ILDA, and ILDA-LiBSVM algorithms . . . . . . . . . . . . . . 100

Table 3.4 Identification accuracy of batch-CCIPCA, ICA, Ind-CCIPCA, ISVM, LDA, LDA-LiBSVM, PCA and PCA-LiBSVM. ..... 101

Table 4.1 Details of the muzzle point image pattern database of cattle . . . . . . . . . . . . . . . . 125

Table 4.2 Identification accuracies of PCA, LDA, ICA, SURF, LBP, and proposed approaches for cattle recognition .......... 129

Table 4.3 Performance of modified appearance-based recognition algorithms such as, Batch-CCIPCA, ICA, IND-CCIPCA, ISVM, LDA, LDA-LiBSVM, PCA, and PCA-LiBSVM . . . . . 130 
Table 4.4 Identification accuracies of Batch-ILDA, CCIPCA-LiBSVM, ICA-LiBSVM, ILDA, and ILDA-LiBSVM algorithms

Table 5.1 Illustration of various texture features extracted from the muzzle point image pattern and their ranges . . . . . . . .

Table 5.2 Summary statistics (mean \pm standard deviation) of few extracted texture features of muzzle pattern of set A, B, and C

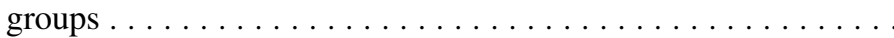

Table 5.3 Classification of cattle based on muzzle point images classes (A, B, and C), each group has different size of muzzle point

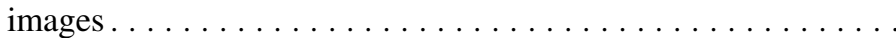

Table 5.4 Classification rate for classes (A, B, and C) groups of muzzle point pattern, each group has different cases (muzzle point

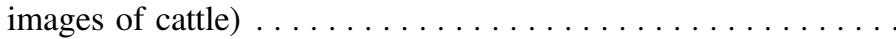

Table 5.5 Recognition rate for (A, B, and C) groups of muzzle point pattern, each group has different cases (muzzle point images

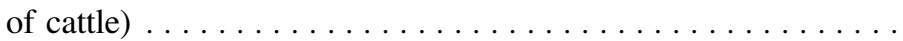

Table 5.6 Classification accuracy rate (\%) for muzzle point image classes (A, B, and C) under occlusion $(\%) \ldots \ldots \ldots \ldots$

Table 5.7 Summary of cattle identification and classification techniques based on muzzle image pattern used in the literatures and experimental results of proposed approach . . . . . . . . . .

Table 6.1 Identification accuracy (\%) of CNN, SDAE, and DBN deep

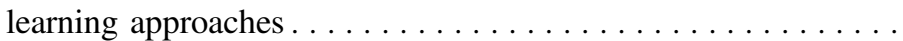

Table 6.2 Identification accuracy (\%) of CNN, SDAE, and DBN deep learning approaches.

Table 6.3 Experimental results are reported in terms of average accuracy with standard deviation over tenfold cross-validation for existing handcrafted texture feature-based methods . . . . . . . .

Table 6.4 Experimental results are reported in terms of average accuracy with standard deviation over tenfold cross-validation for texture holistic feature-based recognition algorithms . . . . . . .

Table 6.5 Experimental results are reported in terms of average accuracy with standard deviation over tenfold cross-validation on holistic feature-based recognition algorithms . . . . . . . . 186

Table 6.6 Comparison of our proposed approach with the literature . . . . 189

Table 7.1 Identification accuracy $(\%)$ of PCA, LDA, ICA,

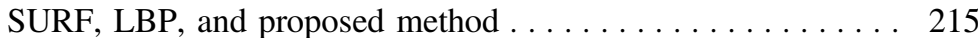

Table 7.2 Identification accuracy of modified version of algorithms . . . . 216

Table 7.3 Identification accuracy of Batch-iLDA, CCIPCA-LiBSVM, ICA-LiBSVM, iLDA, incremental-LDA-LiBSVM

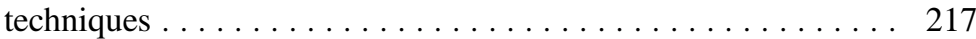

Table 7.4 Recognition time $(\mathrm{s}) \ldots \ldots \ldots \ldots \ldots \ldots \ldots \ldots \ldots \ldots \ldots$

Table 8.1 Current state-of-the-art method for recognition of animal using face biometric modality . . . . . . . . . .

Table 8.2 Animal biometrics database of different species or individual animal . . . . . . . . . . . . . . . 